\title{
SEPARATION OF NONASSOCIATES BY VALUATIONS
}

\author{
DAVID E. BROWN AND MAX D. LARSEN ${ }^{1}$
}

\begin{abstract}
In many classical integral domains, given two nonassociates it is possible to find a valuation on the quotient field of the domain which is nonnegative on the domain and for which the nonassociates have different values. Recent work by Griffin, Harrison, and Manis has extended valuation theory to commutative rings with identity which contain zero divisors. In this paper we investigate the separation of nonassociates by valuations for the extended valuation theory. Our main result states that if $R$ is a ring with a von Neumann regular total quotient ring, then nonassociates can be separated by valuations if and only if there is no unit in the integral closure of $R$ which is not a unit in $R$.
\end{abstract}

All rings considered will be commutative and have identity. A valuation on a ring $K$ is a map $v$ from $K$ onto a totally ordered group with $\infty$ adjoined such that $v(x y)=v(x)+v(y)$ and $v(x+y) \geqq \min \{v(x), v(y)\}$ for all $x, y \in K$. The elements of $K$ at which $v$ is nonnegative is a subring denoted by $R_{v}$ and called the valuation ring of $v$. A subring $R$ of $K$ is a valuation ring with prime ideal $P$ of elements of positive value if and only if for any ring $S$ such that $R \subseteq S \subseteq K$ and prime ideal $M$ of $S$ with $M \cap R=P$ we have $S=R$ [3].

We will restrict ourselves to considering valuations on total quotient rings. Let $R$ be a ring, $K$ its total quotient ring. The following definitions will be useful.

Definition. A collection $\mathscr{V}$ of valuations on $K$ separates nonassociates of $R$ if given any two nonassociates $a, b \in R$, there exists $v \in \mathscr{V}$ such that $v(a) \neq v(b)$.

DEFINITION. If $R$ is a subring of a ring $S$, then $R$ is close to $S$ if every unit of $S$ is also a unit of $R$.

First we restrict ourselves to regular elements.

Presented to the Society, September 1, 1971; received by the editors January 29, 1971 and, in revised form, April 26, 1971.

AMS 1970 subject classifications. Primary 13A15.

Key words and phrases. Separation of nonassociates by valuations, large Jacobson radical, $R$ is close to $S$.

${ }^{1}$ This paper is part of the first author's dissertation at the University of Nebraska. The second author received support during the writing of this paper from the University of Nebraska Research Council in the form of a Senior Faculty Summer Fellowship.

(c) American Mathematical Society 1972 
THEOREM 1. Let $R$ be a ring with total quotient ring $K$ and let $\mathscr{V}$ be the set of all valuations on $K$ which are nonnegative on $R$. Then $\mathscr{V}$ separates regular nonassociates of $R$ if and only if $R$ is close to $\bigcap\left\{R_{v} \mid v \in \mathscr{V}\right\}$.

Proof. If $R$ is not close to $\bigcap\left\{R_{v} \mid v \in \mathscr{V}\right\}$, then there exists a unit $x$ in $\bigcap\left\{R_{v} \mid v \in \mathscr{V}\right\}$ which is not a unit in $R$. Then for all $v \in \mathscr{V}, v(x)+v\left(x^{-1}\right)=$ $v(1)=0, v(x) \geqq 0$, and $v\left(x^{-1}\right) \geqq 0$. Thus if $x=a / b$, then $v(a)=v(b)$ for all $v \in \mathscr{V}$. However $a$ and $b$ are not associates since $x$ is not a unit in $R$.

Conversely, if $R$ is close to $\bigcap\left\{R_{v} \mid v \in \mathscr{V}\right\}$ and if $a, b$ are regular elements of $R$ with $v(a)=v(b)$ for all $v \in \mathscr{V}$, then $v(a / b)=v(b / a)=0$ for all $v \in \mathscr{V}$. Hence $a / b=u$ is a unit in $\bigcap\left\{R_{v} \mid v \in \mathscr{V}\right\}$, and so is a unit in $R$. Thus $a$ and $b$ are associates.

Separation of nonassociates by valuations on a field is completely determined by the following corollary.

COROLlary. If $R$ is an integral domain with quotient field $K$, then the set $\mathscr{V}$ of all valuations on $K$ which are nonnegative on $R$ separates nonassociates of $R$ if and only if $R$ is close to its integral closure.

If two elements differ by a nilpotent, then they cannot be separated by valuations. For let $x \in R$ be nilpotent and let $a$ be any element of $R$. If $b=a+x$, then $(b-a)^{n}=0$ for some $n$, so $v(b-a)=\infty$ for all valuations $v$ which are nonnegative on $R$. Hence

$$
v(b)=v((b-a)+a) \geqq \min \{v(b-a), v(a)\}=v(a) .
$$

But similarly $v(a) \geqq v(b)$, so $v(a)=v(b)$.

On the other hand, the next result gives some information about elements which cannot be separated by valuations.

THEOREM 2. If $a, b$ are elements of $a$ ring $R$ such that $v(a)=v(b)$ for all valuations $v$ which are nonnegative on $R$, then $a^{n}=b r$ for some integer $n$ and some $r \in R$.

Proof. If there were a prime ideal $P$ of $R$ such that $a \in P, b \notin P$, then there would exist a valuation pair $(S, M)$ with $R \subseteq S, M \cap R=P, a \in M$, and $b \notin M$. Then $v(a)>0$ and $v(b)=0$ where $v$ is the valuation of $(S, M)$. Hence $a$ and $b$ belong to precisely the same prime ideals of $R$. Thus $a$ belongs to the radical of $b R$; hence the result.

COROLlARY. If $a, b$ are elements of a ring $R$ such that $v(a)=v(b)$ for all valuations $v$ which are nonnegative on $R$, then either $a$ and $b$ are both regular or are both zero divisors.

As unsatisfactory as Theorem 2 is, the following example shows that in general it cannot be improved. Let $Z$ denote the integers, $Q$ the rationals, 
$Z_{p}$ the integers modulo $p$, and $Z_{(p)}$ the $p$-adic integers. Set

$$
K=Q \oplus Z_{4} \oplus Z_{6} \oplus Z_{8} .
$$

Then $K$ is its own total quotient ring. Fix a prime integer $p$. Let

and

$$
R=\left\{(a, b, c, d) \in K \mid a \in Z_{(p)}\right\}
$$

$$
P=\left\{(a, b, c, d) \in K \mid a \in(p) Z_{(p)}\right\} .
$$

Then $(R, P)$ is a valuation pair. If $x=(p, 2,4,0)$ and $y=(p, 0,2,2)$, then $x^{6}=y^{6}$, so $x$ and $y$ have the same value for all valuations which are nonnegative on $R$. Also $x^{2}=y(p, 1,2,0)$ and $y^{2}=x(p, 2,1,2)$, but there do not exist $r$ or $s$ such that $x=y s$ or $y=x r$, nor do there exist units $u$ or $w$ such that $x^{n}=y u$ or $y^{n}=x w$ for any integer $n$.

In the discussion following the corollary to Theorem 1, it was shown that $x-y$ nilpotent means $v(x)=v(y)$ for all valuations $v$ which are nonnegative on $R$. Note that in the above example, $x-y$ is not nilpotent.

Henceforth $R$ will be a ring with total quotient ring $K, J$ will be the Jacobson radical of $K$, and $\mathscr{V}$ will denote the set of all valuations on $K$ which are nonnegative on $R$. We are interested in determining necessary and/or sufficient conditions for $\mathscr{V}$ to separate nonassociates of $R$.

A ring $K$ is von Neumann regular if for every $a \in K$ there exists $x \in K$ such that $a^{2} x=a$.

It is well known that if $K$ is von Neumann regular then $J=0$, and $K$ is a total quotient ring. An additional fact will make explicit the class of rings that will be discussed in the theorem below.

Proposition [2, p.55]. A ring $K$ with no nonzero nilpotents is von Neumann regular if and only if prime ideals of $K$ are maximal.

THEOREM 3. If $R$ is a ring with a von Neumann regular total quotient ring $K$, then $\mathscr{V}$ separates nonassociates of $R$ if and only if $R$ is close to its integral closure.

Proof. Since $K$ is von Neumann regular, the integral closure of $R$ is $\bigcap\left\{R_{v} \mid v \in \mathscr{V}\right\}$ [1, Proposition 9]. Thus Theorem 1 asserts the necessity of $R$ being close to its integral closure.

Now suppose $R$ is close to its integral closure and that for $a, b \in R$, $v(a)=v(b)$ for all $v \in \mathscr{V}$. Then from Theorem $2, a^{n}=b r$ for some integer $n$ and some $r \in R$. Since $K$ is von Neumann regular there exists $s \in K$ such that $(r a)^{2} s=r a$ and so $r a(r a s-1)=0$. Let $P$ be a prime ideal of $K$; then either $r a \in P$ and ras $-1 \notin P$, or $r a \notin P$ and ras $-1 \in P$. Thus $r a+$ ras -1 is a unit in $K$, and since $b \notin P$ implies $a \notin P$ and $r \notin P$, it follows that

$$
b(\text { ras }-1) \in J=0 .
$$

Hence $a^{n+1}=b(r a+r a s-1)$ and $a u=b$ where $u=a^{n}(r a+r a s-1)^{-1}$. 
Again because $K$ is von Neumann regular there exists $t \in K$ such that $u(u t-1)=0$. Since $u(u t-1)=0$, we have $a^{n}(u t-1)=0$ and that $a(u t-1) \in$ $J=0$, so $a(u+u t-1)=b$. Arguing as above, it is clear that $u+u t-1$ is a unit in $K$.

Let $v \in \mathscr{V}$; if $v(a)=v(b)<\infty$, then $v(u+u t-1)=0$. Suppose that $v(a)=$ $v(b)=\infty$; then $v(r a+r a s-1)=\min \{v(a(r+r s)), v(1)\}=v(1)=0$ and $v(u)=$ $n v(a)-v(r a+r a s-1)=\infty$. Hence $v(u+u t-1)=\min \{v(u(1+t)), v(1)\}=$ $v(1)=0$. We have shown that $v(u+u t-1)=0$ for all $v \in \mathscr{V}$; hence $u+u t-1$ is a unit in $R$. This proves that $a$ and $b$ are associates in $R$.

Most of the rest of this paper is devoted to examining the strength of this theorem. First we give an example to show that the converse is not true. The method of constructing the example is due to Malcolm Griffin $\left[2\right.$, p. 34]. Let $A$ be the algebraic closure of $Q$. For each $a \in A$, let $\psi_{a}$ be the epimorphism from $Q[X]$ onto $Q(a)$ defined by $\psi_{a}(f(X))=f(a)$. Let $I=A \times N$ where $N$ is the set of positive integers. For $i=(a, n) \in I$, set $K_{i}=Q(a)$ and define $\psi$ from $Q[X]$ onto the direct product of the $K_{i}$ by $\pi_{i}(\psi(f(X)))=\psi_{a}(f(X))$ where $i=(a, n)$ and $\pi_{i}$ is the $i$ th projection. Set $B=\psi(Q[X]), F=\oplus \sum K_{i}$, and $K=B \oplus F$.

If $b+y$ is a regular element of $K$ for $b \in B, y \in F$, then there exists $f(X) \in Q[X]$ such that $b=\psi(\cdot f(X))$. One can show that $f(X)=c$ is a nonzero rational and that $b^{-1}=\psi\left(c^{-1}\right) \in K$. Thus $b+y$ has $b^{-1}-b^{-1} y /(b+y)$ as inverse in $K$, so $K$ is a total quotient ring.

Choose $i=(a, n) \in I$. If $a \in Z$, let $\mathscr{V}_{i}^{\prime}$ be the set of $p$-adic valuations on $Q$. If $a \in A / Z$, let $\mathscr{V}_{i}^{\prime}$ be the set whose only member is the trivial valuation on $K_{i}$. For each $i \in I$ and $v_{i}^{\prime} \in \mathscr{V}_{i}^{\prime}$ define a valuation $v_{i}$ on $K$ by $v_{i}(k)=v_{i}^{\prime}\left(\pi_{i}(k)\right)$ for all $k \in K$. Let $\mathscr{V}$ be the set of all valuations obtained in this manner. For $v \in \mathscr{V}, R_{v}=\left\{k \in K \mid \pi_{i}(k) \in R_{v_{i}}{ }\right\}$ for some $v_{i}^{\prime}$ in some $\mathscr{V}_{i}^{\prime}$. Let

$$
R=\bigcap\left\{R_{v} \mid v \in \mathscr{V}\right\}
$$

clearly $R$ is integrally closed and $\psi(Z[X]) \subseteq R$. One can show that $K$ is the total quotient ring of $R$.

Let $x, z$ be elements of $R$ with $v(w)=v(z)$ for all $v \in \mathscr{V}$. Then $w=b+y$ and $z=s+t$ where $b=\psi(f(X)), s=\psi(g(X))$, and $y, t \in F$. For fixed $m \in Z$ and for infinitely many $i=(m, n) \in I, \pi_{i}(w)= \pm \pi_{i}(z)$ since $\mathscr{V}_{i}^{\prime}$ separates nonassociates in $\pi_{i}(R)$. This implies that for each integer $m,|f(m)|=|g(m)|$ and thus $f(X)=e g(X)$ where $e= \pm 1$. If $\pi_{i}(y) \neq 0$ or $\pi_{i}(t) \neq 0$, then $\pi_{i}(w)=$ $e_{i} \pi_{i}(z)$ for some unit $e_{i} \in \pi_{i}(R)$. Now define $u$ by $\pi_{i}(u)=e_{i}$ if $\pi_{i}(y) \neq 0$ or $\pi_{i}(t) \neq 0$, and by $\pi_{i}(u)=e$ otherwise. Then $w=u z$ and it can be verified that $u$ is a unit in $R$. Furthermore $K$ has no nonzero nilpotent elements. However, since $K / F \cong Q[X], K$ contains a nonmaximal prime ideal, and hence $K$ is not von Neumann regular. 
A ring $K$ is said to have large Jacobson radical if prime ideals of $K / J$ are maximal. Thus the proposition preceding Theorem 3 indicates that a total quotient ring $K$ is von Neumann regular if and only if $J$ is both large and zero. Examples will be given to show that neither $J$ large nor $J=0$ is sufficient for the equivalence of $\mathscr{V}$ separates nonassociates of $R$ and $R$ is close to its integral closure.

Consider a subdirect product of fields, each of characteristic 0 , which contains their direct sum. Let $K$ be the subring generated by their direct sum and 1. Then $K$ is a von Neumann regular ring, and so if $R$ is any subring of $K$ whose total quotient ring is $K$, then the valuations on $K$ which are nonnegative on $R$ separate nonassociates of $R$ if and only if $R$ is close to its integral closure.

The example below shows that it is essential that the fields be of characteristic 0 . The example is a ring $K$ which is a subdirect product of fields with subring $R$ for which the valuations on $K$ which are nonnegative on $R$ do not separate nonassociates of $R$.

Let $H$ denote the set of prime positive integers, $N$ the positive integers, and $I=H \times N$. For $p \in H$, let $f_{p}: Z \rightarrow Z /(p)$ be the canonical homomorphism and for $i=(p, n) \in I$, let $K_{i}=Z /(p)$. Define $f$ from $Z$ to the direct product of the $K_{i}$ by $\pi_{i}(f(x))=f_{p}(x)$ for all $x \in Z$ where $i=(p, n)$. Set $B=f(Z)$, $F=\oplus \sum\left\{K_{i} \mid i \in I\right\}$, and $K=B \oplus F$. Then $K$ is a total quotient ring, and is of the described form. The only valuations on $K$ are trivial and correspond to the prime ideals $F, f((p))+F$, and $P_{i}=\left\{a \in K \mid \pi_{i}(a)=0\right\}$. The elements $a, b$ of $K$ defined by $\pi_{i}(a)=f_{p}(2)$ and $\pi_{i}(b)=f_{p}(4)$ for $i=(p, n)$ belong to exactly the same prime ideals of $K$, and so have the same value for all valuations on $K$. Clearly $a$ and $b$ are not associates. Furthermore $K$ is integrally closed.

This example can be modified in order to have a ring with nontrivial valuations which are not adequate to separate nonassociates. The ring $R=Z \oplus K$ has $Q \oplus K$ as a total quotient ring, where $Q$ denotes the rational numbers. The valuations on $Q \oplus K$ are the extensions of the valuations on $Q$ and $K$; hence the elements $(r, a)$ and $(r, b)$, where $r \in Z$, are nonassociates in $R$ having the same value for all valuations on $Q \oplus K$.

In either case above $J=0$, so this provides the example to show that $J=0$ is not sufficient for the conclusion of Theorem 3 .

The next result shows that under certain restrictions $J=0$ is necessary for the separation of nonassociates. We do not know if this is true in general. In all examples we have seen, $J=0$ if nonassociates are separated by valuations. Let $R$ be a ring and express its total quotient ring $K$ as a subdirect product of subdirectly irreducible rings $K_{\lambda}, \lambda \in \Lambda$. Suppose $K$ contains the direct sum of the $K_{\lambda}$. 
THEOREM 4. If $R$ is as described above and $\mathscr{V}$ separates nonassociates of $R$, then $J=0$.

Proof. Suppose $J \neq 0$; then there exists $\mu \in \Lambda$ such that $K_{\mu}$ contains a nonzero nilpotent element $x_{\mu}$, say $\left(x_{\mu}\right)^{n}=0$. Define $x$ by $\pi_{\mu}(x)=x_{\mu}$ and $\pi_{\lambda}(x)=0$ for $\lambda \neq \mu$. Write $x=c / d$ for $c, d \in R, d$ regular and $x_{\mu}=c_{\mu} / d_{\mu}$, $c_{\mu}, d_{\mu} \in K_{\mu}, d_{\mu}$ regular. Then $\pi_{\mu}(c)=c_{\mu}$ and $\pi_{\lambda}(c)=0$ if $\lambda \neq \mu$, so $c^{n}=0$. The element $t \in K$ defined by $\pi_{\mu}(t)=1$ and $\pi_{\lambda}(t)=0$ if $\lambda \neq \mu$ can be written as $a / r$ for some $a, r \in R, r$ regular. Thus $\pi_{\mu}(a)=0$ and $\pi_{\lambda}(a)$ is a regular element of $K_{\lambda}$ for $\lambda \neq \mu$.

Let $b=a+c$, then by the remark following the corollary to Theorem 1 , $v(a)=v(b)$ for all $v \in \mathscr{V}$. Therefore there is a unit $u$ of $R$ such that $a u=b$. Then $\pi_{\mu}(u)$ is a unit in $K_{\mu}$ and $0=\pi_{\mu}(u) \pi_{\mu}(a)=\pi_{\mu}(b) \neq 0$. This contradiction shows that $J=0$.

Now we give an example to show that $K$ having large Jacobson radical cannot be used to replace $K$ being von Neumann regular in Theorem 3 . Let $F$ be the field of 2 elements. For each positive integer $k$, let $D_{k}$ be the

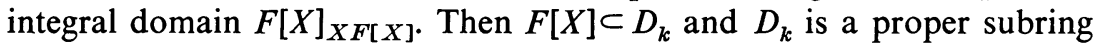
of both $F[[X]]$ and $F(X)$. For each $k$, let $P_{k}=X F[X]_{X F[X]}$ and let $K$ be the subring of the direct product of the $D_{k}$ generated by $P=\oplus \sum P_{k}$ and 1 . Then $K$ is a total quotient ring. Let $R$ be the subring of $K$ generated by 1 and $\oplus \sum R_{k}$ where $R_{k}=X F[X]$ for all $k$. Then $K$ is the total quotient ring of $R$ and the nonassociates $(\cdots, 0, X, 0, \cdots)$ and $\left(\cdots, 0, X^{2}, 0, \cdots\right)$ cannot be separated by valuations on $K$ which are nonnegative on $R$ even though $R$ is integrally closed. Furthermore $P=J$ and $K$ has large Jacobson radical since $P$ is the unique maximal ideal of $K$.

Our final result is a partial generalization of Theorem 3 to rings with nilpotent elements. By $\mathscr{V}$ separates nonassociates of $R$ to within a nilpotent we will mean that for $a, b \in R$ such that $v(a)=v(b)$ for all $v \in \mathscr{V}, a=b u+n$ where $u$ is a unit in $R$ and $n \in R$ is nilpotent.

THEOREM 5. Let $R$ be a ring whose total quotient ring has large Jacobson radical and $J=N$, the ideal of nilpotents of $K$. Then $\mathscr{V}$ separates nonassociates of $R$ to within a nilpotent if $R$ is close to its integral closure.

Proof. Since $K$ has large Jacobson radical, the integral closure of $R$ is $R^{*}=\bigcap\left\{R_{v} \mid v \in \mathscr{V}\right\}$ [2, p. 44]. Also note that since for each $v \in \mathscr{V}$, $J=N \subset R_{v}$, we have $N \subset R^{*}$. In view of the proposition preceding Theorem $3, K / J$ is a von Neumann regular ring. Furthermore since $N=J$, the valuations on $K / J$ are exactly those induced by the valuations on $K$; this makes $R^{*} / J$ an integrally closed subring of $K / J$.

Let $a, b \in R$ be such that $v(a)=v(b)$ for all $v \in \mathscr{V}$, and denote the images of $a, b$ in $R^{*} / J$ by $a^{\prime}, b^{\prime}$, respectively. Thus there exists $u \in R^{*}$ such that 
its image $u^{\prime}$ is a unit in $R^{*} / J$ and $a^{\prime}=b^{\prime} u^{\prime}$. Now $v(u)=0=v(1)$ for all $v \in \mathscr{V}$ so by the corollary to Theorem $2, u$ is not a zero divisor in $R^{*}$. It follows that $a-b u$ is a nilpotent in $R$ which proves the theorem.

\section{BIBLIOGRAPHY}

1. Malcolm Griffin, Prüfer rings with zero divisors, J. Reine Angew. Math. 239/240 (1969), 55-67. MR 41 \#188.

2. - Valuation theory and multiplication rings, Queen's University Preprint \#1970-37.

3. Merle E. Manis, Valuations on a commutative ring, Proc. Amer. Math. Soc. 20 (1969), 193-198. MR 38 \#2134.

Department of Mathematics, University of Nebraska, Lincoln, Nebraska 68508

Current address (Brown): Department of Mathematics, Stephens College, Columbia, Missouri 65201 